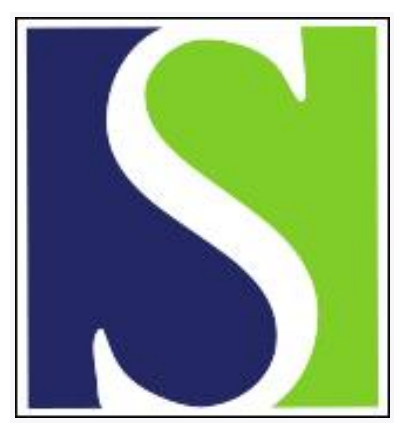

Scand J Work Environ Health 1986;12(4):407-412

https://doi.org/10.5271/sjweh.2122

Issue date: Aug 1986

Assessment of vibration levels associated with hand-held roadbreakers.

by Tasker EG

This article in PubMed: www.ncbi.nlm.nih.gov/pubmed/3775330

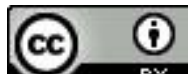

This work is licensed under a Creative Commons Attribution 4.0 International License 


\title{
Assessment of vibration levels associated with hand-held roadbreakers
}

\author{
by Eric G Tasker, BSc, MSc, CEng, MIGasE ${ }^{1}$
}

\begin{abstract}
TASKER EG. Assessment of vibration levels associated with hand-held roadbreakers. Scand J Work Environ Health 12 (1986) 407-412. The hand-held roadbreaker plays a vital role in enabling the British gas distribution workforce to install gas supply pipework. Hence, any potential problem which could lead to a restriction of the use of this equipment would present pipework installation departments with serious operational problems. A test program was therefore set up to enable risks to operators to be quantified. The vibration levels measured exceeded proposed limits laid down in current guidelines for vibration exposure. If these limits were realistic, a high prevalence of vibration-induced white finger (VWF) would have been expected. However, a survey of the workforce using the roadbreakers indicated a VWF prevalence of 20 times less than that predicted by the guidelines. It was concluded that a possible explanation could be that the current frequency-weighting factors used in the guidelines are inappropriate for application to this type of equipment with dominant frequencies below $25 \mathrm{~Hz}$. The application of the current weighting factors to the test data produced during the program led to high weighted acceleration values and unrealistic dose-response relationships. It is proposed that the application of the various guidelines to the use of this equipment be reconsidered.
\end{abstract}

Key terms: British gas meter readers, hand grip, labor force, laser.

The work of the British gas distribution labor force involves excavating through road and pedestrian access areas in order to repair and extend the gas distribution pipework system. For this work, power tools are required, the hand-held roadbreaker being an essential item of equipment used during the course of this activity.

Alternative machine-mounted items of equipment are under development which could reduce the extent to which hand-held tools are required, but for the foreseeable future hand tools will predominate.

These hand tools are percussive in operation and give vibration and shock load feedback to their operators. In view of the known relationships between shock and vibration loads and the incidence of associated disorders, including vibration-induced white finger (VWF), among operators of vibrating tools, the risk to British gas workers needed to be quantified.

A program of vibration measurement was carried out which included a representative sample of pneumatically and hydraulically powered hand-held roadbreakers working in a typical operational environment. During this program a range of vibration measurement methods was evaluated, including the development of acceptable clamp arrangements and associated accelerometers in order that repeatable and representative data could be recorded. Attempts were also made to use a laser vibrometer system to measure vibration

' British Gas Corporation, Newcastle upon Tyne, England.

Reprint requests to: Mr EG Tasker, British Gas Corporation, Research and Development Division, Engineering Research Station, Killingworth, PO Box ILH, Newcastle upon Tyne, NE99 1LH, England. levels realized on the surface of the operator's hands and compare these results with the more conventional measurement methods employed.

\section{Test methods}

The measurement of hand vibration levels on roadbreakers presents some special problems with regard to the selection of suitable instrumentation. In addition careful attention must be given to the methods employed to mount the transducers used to carry out the measurements.

The first series of tests was carried out with the accelerometer and mechanical filter assembly mounted directly on the roadbreaker handle. A simple clamp arrangement was used, and the mounted assembly is shown in figure 1 .

When the breaker is fitted with soft rubber or plastic handles, some problems arise when a clamping technique is used to mount the accelerometer assembly on the handle. The degree of coupling with soft rubber handles is dependent on the grip pressure applied by the operator, and it is difficult to determine the degree to which a clamp must be tightened to simulate this particular grip pressure. The effects of a tight and loose clamp are shown in figure 2 , which shows good agreement at the lower end of the spectrum, but less agreement at the high-frequency end, as a loose mount inhibits the transfer of high frequencies. The poor agreement around the band centered at $50 \mathrm{~Hz}$ is difficult to explain but is probably due to natural resonance effects around this point. 
For more meaningful and consistent data over the entire measurement spectrum on roadbreakers fitted with soft rubber handle grips, it is possible to use a different measurement technique. The accelerometer

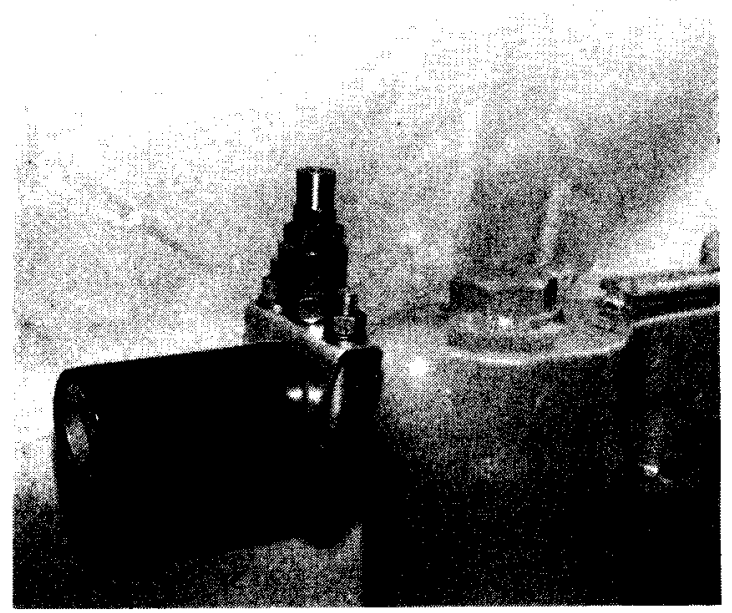

Figure 1. Mechanical filter and clamping system of a piezoelectric accelerometer. is mounted into a special adaptor which is gripped between the operator's hand and the roadbreaker handle. This method should realistically reflect the used grip strength on the soft handle. Bruel \& Kjaer have developed such a system based on a hand grip (WA 0374) and a miniature accelerometer (4374). Figure 3 illustrates the system components and the operating position on a roadbreaker. Figure 2 compares the response of the hand-grip system with that of a clamped system. There is good agreement at the lower end of the spectrum, but towards the high-frequency end the hand-gripped accelerometer gives a somewhat higher response.

The measurement techniques previously described either assume that vibration generated on the breaker handle is transferred to the operator's hand or rely on a device gripped between the operator's hand and the breaker handle to indicate the level of vibration being transferred to the hand. A possibly more accurate method of assessing the vibration levels in the operator's hand would be to measure the vibration level on say a knuckle joint as the roadbreaker is operated.

The best approach would be to use a noncontact method of measuring the vibration levels, and this approach can be carried out with the use of a laser vib-

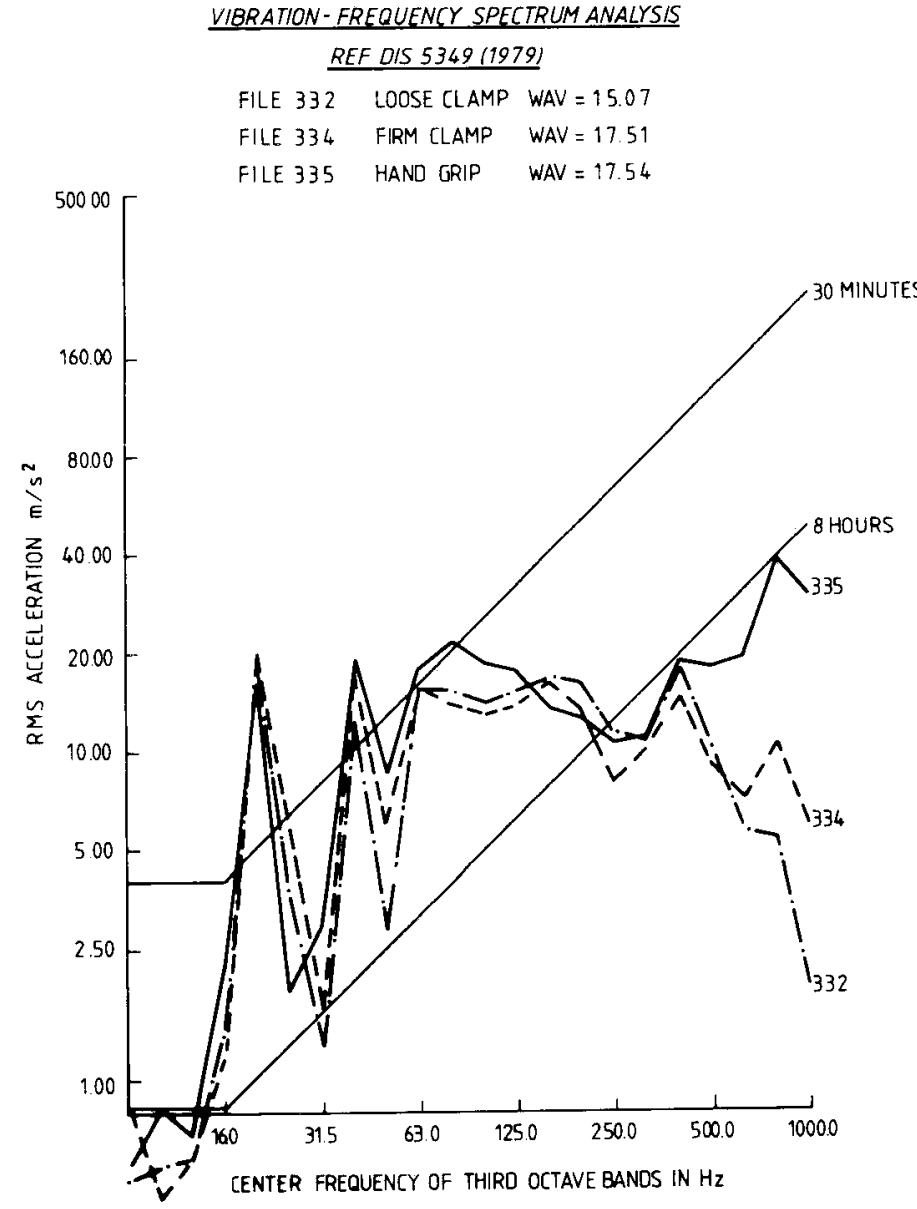

Figure 2. Frequency response for a range of accelerometer mountings. (WAV = weighted acceleration value). 


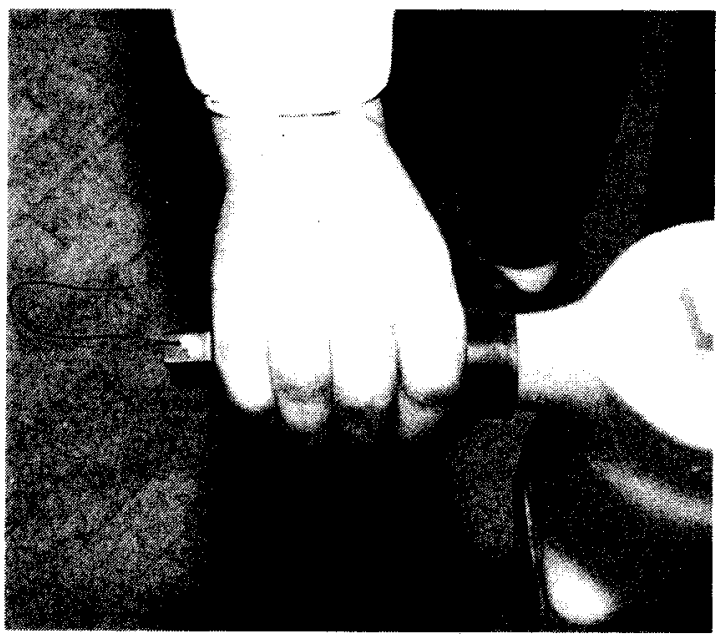

Figure 3. Hand grip and accelerometer in working position.

rometer, although this method can be affected by skin resonances. Figures 4 and 5 show a Disa laser vibrometer system in use to measure the vibration levels on an operator's knuckle joint. The laser beam is directed onto a small reflective tape target attached to the operator's knuckle. Unlike an accelerometer the tape target has a very low mass and therefore gives good coupling to the knuckle joint through the taut skin above.

The operator has to keep the beam, which appears as an intense red spot, aligned on the target on his knuckle, and this action can be difficult during the use of a powerful breaker with the steel in a free condition. The laser beam can cause eye damage if viewed directly for a length of time, and the operator must wear suitable eye protection. The test site should also be screened to prevent the beam from entering other areas. Figure 6 compares velocities measured on the operator's knuckle with the laser system to velocities measured with an accelerometer/filter assembly mounted on the breaker handle. The data compare satisfactorily over the frequency spectrum and therefore suggest that a handle-mounted accelerometer/filter assembly does give an acceptable measure of the vibration transmitted to the operator's hand over the frequency range of importance.

\section{Test results}

The test data are presented in the form of acceleration against frequency graphs, and frequency-weighted acceleration values have been calculated based upon the weighting network defined in ISO/DIS 5349 (2). Taking a typical value of weighted acceleration and then converting it to a 4-h energy equivalent acceleration value for $0.5-\mathrm{h}, 1.0-\mathrm{h}$, and 2 -h exposure gives the 4-h energy equivalent acceleration figures of $6.2,8.8$, and 12.5, respectively. According to the dose-response

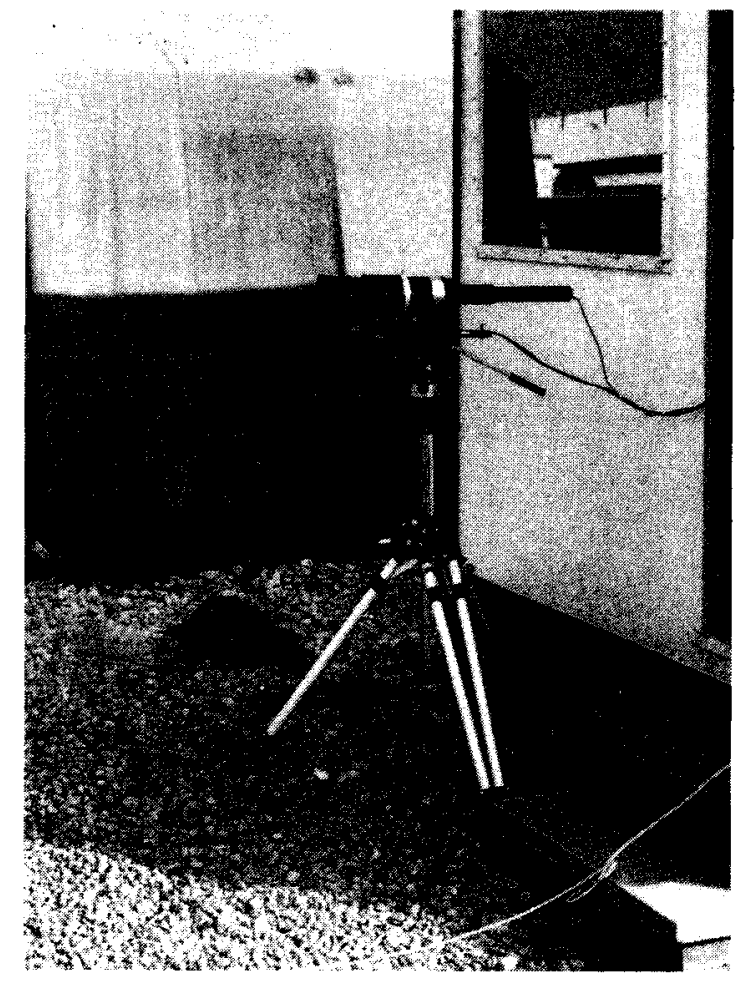

Figure 4. Disa $55 \mathrm{X}$ laser vibrometer.

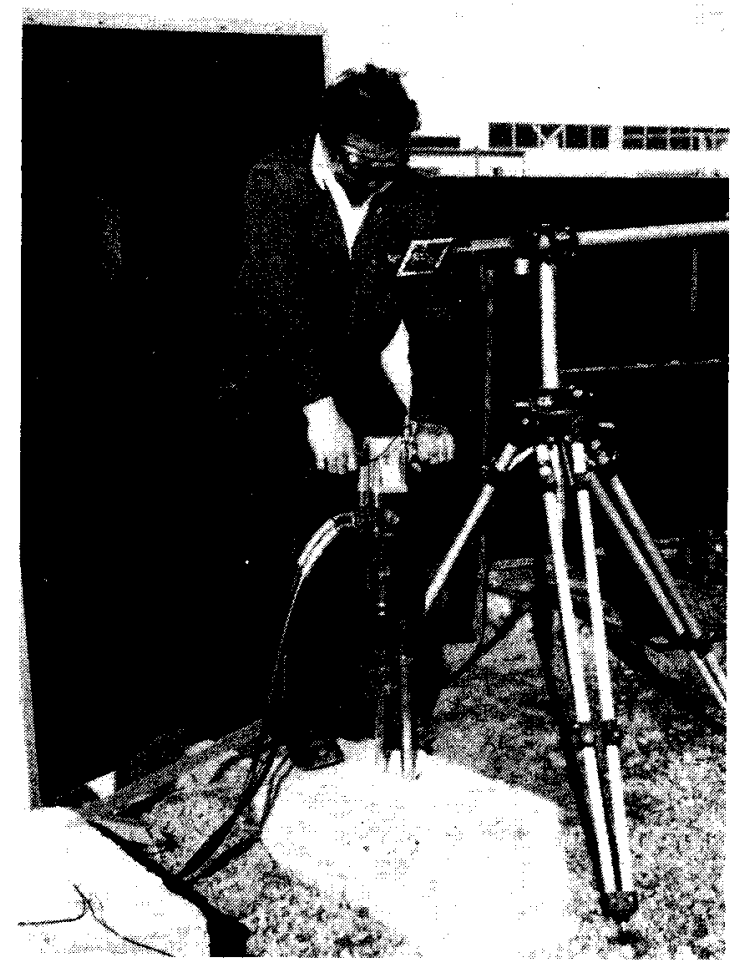

Figure 5. Laser reflector and mounting system. 


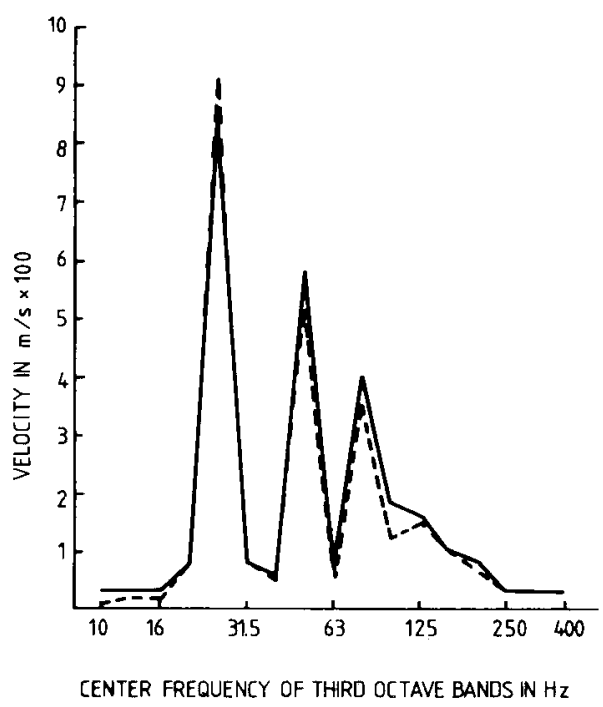

Figure 6. Comparison between the results of the laser system $(-)$ and those of the clamped piezoelectric accelerometer (- - ).

relationships developed in ISO/DIS 5349, the following observations can be made:

1. With a weighted acceleration of 6.2 , in six years of vibration exposure, $10 \%$ of the working population would be suffering from finger blanching associated with vibration exposure. After 10 years, up to 25 $\%$ of the population would at least be suffering from finger blanching with an undefined proportion suffering from the more advanced effects of VWF.

2. With a weighted acceleration of 8.8 , in four years of exposure, $10 \%$ of the working population would be suffering from finger blanching, and, after 10 years, up to $50 \%$ of the population would at least be suffering from finger blanching, again with an undefined proportion with advanced symptoms.

3. With a weighted acceleration of 12.5 , in 2.5 years of exposure, $10 \%$ of the population would be suffering from finger blanching, and, after 10 years, in excess of $50 \%$ could be suffering from advanced symptoms.

These predictions severely conflict with the findings of a recent survey of 895 British gas workers (3) who use vibrating tools, principally roadbreakers, on a regular basis with exposure times varying from $0.5 \mathrm{~h}$ to $2 \mathrm{~h}$ per day. In this survey the reference population was composed of 553 meter readers who did not use vibrating tools but who operated in an environment similar to that of the exposed group. The prevalence of white finger was $9.6 \%$ in the exposed group compared with $9.5 \%$ in the meter readers' group $(12.2 \%$ in the former group after standardization for age differences), and the difference from that of the reference group was not statistically significant. A statisti- cally significant association was found between the prevalence of white finger and age for both the distribution workers and the meter readers $(p<0.01$ and $p<0.05$, respectively). Within the group exposed to vibrating tools in the gas industry only, no direct association was found between the prevalence of white finger and years of vibrating tool use.

In the opinion of the authors there was no epidemiologic evidence to suggest that distribution workers who work with vibrating tools in the gas industry are more liable to develop symptoms of white finger than meter readers/collectors who do not work with vibrating tools. It appeared, therefore, that VWF was not a particular problem in the gas industry among men who use vibrating tools to break or reinstate road surfaces.

The guidelines in ISO/DIS 5349 therefore appear to overestimate the risks associated with roadbreakers, at least in relation to the onset of white finger symptoms. There are several possible reasons for this apparent disagreement, and they are presented in the following discussion.

1. The relatively short exposure time of less than 2 $\mathrm{h} / \mathrm{d}$ may be insufficient to allow VWF symptoms to develop due to some daily use/recovery time phenomenon which influences the predictions.

2. The method of gripping the tools may have some influence on the predictions since, with roadbreakers, the tool is not firmly gripped. Most of the epidemiologic data relates to operators using equipment requiring a firm hand grip to operate and support the tool. This necessity may influence the effective weighted acceleration value.

3. The third and most important factor could be that the frequency weighting curve proposed in ISO/DIS 5349 is not the appropriate one to apply to tools and equipment with dominant frequencies below $25 \mathrm{~Hz}$. The credibility of the weighting curve has only been supported to date by data obtained from tools and equipment with dominant frequencies well in excess of $25 \mathrm{~Hz}$.

The exposure times may be insufficient to allow the development of VWF symptoms in the workforce, although high levels have been reported by Chatterjee et al (1) when tools with higher dominant frequencies were used for similar exposure times. The exposure times are typical of current operational practices in the British gas industry, but they could change in the future with a greater dependence on a mechanized plant and a potential reduction in exposure time.

A noncontact method of vibration assessment with the laser vibrometer discussed earlier has been used, and the data obtained have been compared to those obtained with a piezoelectric accelerometer clamped to the roadbreaker handle. It was shown that, over the 
critical low-frequency end of the spectrum, the data retrieved from both methods are similar; hence the grip force does not appear in this case to be an important factor in quantifying these low-frequency vibration levels.

The final point on the relevance of the proposed frequency-weighting curve is a key area since the weighted values obtained for roadbreakers lead to high figures and hence unrealistic dose-response relationships in the experience of the British Gas Corporation presented in reference 2 .

\section{Proposed frequency-weighting relationship}

In order for some agreement to be obtained between the findings discussed by Walker et al (3), the predicted incidence of VWF in ISO/DIS 5349, and the frequency-weighted acceleration values obtained during the testing of roadbreaking equipment, a revised table of frequency-weighting factors is proposed. This proposed table of weighting factors originates from the same source as the ISO/DIS 5349 factors but has been modified at the low-frequency end to reduce the effect on the final weighted acceleration value of the attenuation factors in the frequency range $10-25 \mathrm{~Hz}$. The proposed weighting curve is plotted together with the ISO/DIS 5349 curve in figure 7.

Again with the use of a typical value of weighted acceleration, this time using the modified weighting factors, the following 4-h equivalent acceleration values (EAV) for the daily exposure times indicated are:

$$
\begin{aligned}
& 30 \text { min EAV 4-h } 1.57 \\
& 1 \mathrm{~h} \quad \text { EAV 4-h } 2.24 \\
& 2 \mathrm{~h} \text { EAV 4-h } 3.17
\end{aligned}
$$

With these revised frequency-weighted acceleration figures applied to the dose-response relationship defined in ISO/DIS 5349, the risks associated with the use of hand-held roadbreakers and the incidence of VWF can be realistically predicted so that closer agreement with the findings of Walker et al (3) is allowed for.

This revised weighting curve therefore appears to be more appropriate for hand-held roadbreakers with the type of low-frequency characteristics measured during this test program.

\section{Discussion}

The measurement methods employed during the course of this evaluation program all appear to give repeatable and comparable results with solid metal handles or with a thin layer of insulating material between the hand grip and the steel handle. With thicker layers of insulating material, the clamp pressure applied to the accelerometer mounting becomes important, and under these circumstances either the laser method or the

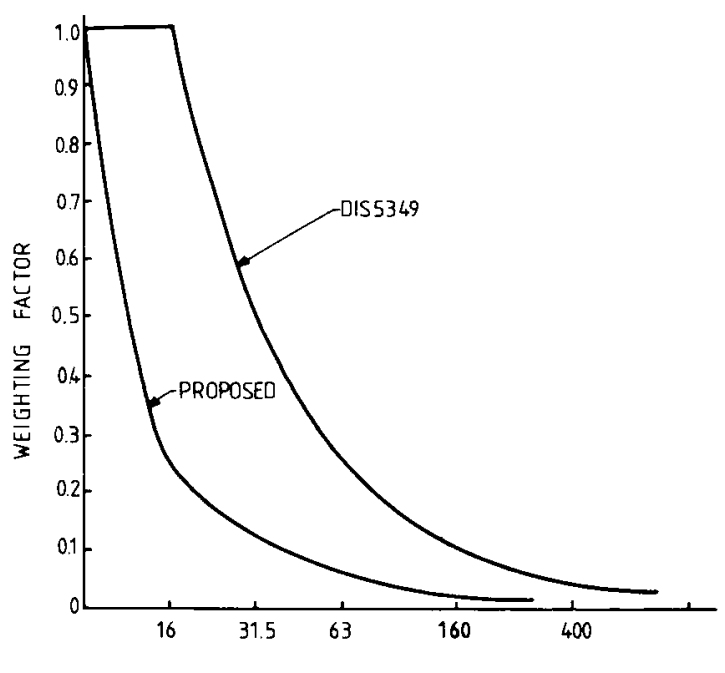

CENTER FREQUENCY OF THIRD DCTAVE BANDS IN Hz

Figure 7. The proposed weighting curve and that of ISO/DIS 5349 (2).

use of a hand-grip mounting would be more appropriate.

Since, however, the low-frequency end of the vibration frequency spectrum is the most critical during the calculation of weighted acceleration values, no matter what measurement method is employed and no matter what hand-grip materials are used, representative values of weighted acceleration have been obtained. Hence all measurement methods appear to portray similar peak acceleration values below $75 \mathrm{~Hz}$.

\section{Conclusions}

1. The conventional piezoelectric accelerometer, when clamped to the tool handles or secured to a hand-grip mounting, allows realistic assessments of the vibration levels over the low-frequency end of the spectrum, which influences the weighted acceleration values.

2. The laser vibrometer does not appear to offer a significant measurement advantage, when the cost of this equipment and the difficulty in setting up the device under normal working conditions is considered.

3. The proposed modified weighting curve described in this paper appears to be a realistic method of assessing the risk to operators of roadbreakers in comparison to that specified in ISO/DIS 5349.

\section{Acknowledgments}

The author wishes to thank his colleagues at the Engineering Research Station, in particular Mr JJ Roff for his invaluable assistance in carrying out the vibration measurement work associated with this paper. 
The author would also like to thank the British Gas Corporation for permission to publish this paper.

\section{References}

1. Chatterjee DS, Petrie A, Taylor W. Prevalence of vibration induced white finger in fluorospar mines in Wear- dale. Br J Ind Med 35 (1978) 208-218.

2. International Organization for Standardization. Guide for the measurement and the assessment of human exposure to vibration transmitted to the hand. Geneva 1983. (ISO/ DIS 5349).

3. Walker DD, Jones B, Ogston S, Tasker EG, Robinson AJ. A study of white finger in the Gas Industry. $\mathrm{Br} \mathrm{J}$ Ind Med 42 (1985) 672-677. 